\title{
Portable Pulse Meter with Simple Design
}

\author{
Tian-Syung Lan, ${ }^{1,2 *}$ Yan Jiao, ${ }^{1}$ Xuan-Jun Dai, ${ }^{* * *}$ \\ Ho-Sheng Chen, ${ }^{3}$ and Zhingqing Yang ${ }^{4}$ \\ ${ }^{1}$ College of Mechanical and Control Engineering, Guilin University of Technology, Guangxi 541004, China \\ ${ }^{2}$ Department of Information Management, Yu Da University of Science and Technology, \\ Miaoli County 36143, Taiwan \\ ${ }^{3}$ College of Mechanical and Electrical Engineering, Guangdong University of Petrochemical Technology, \\ Maoming Guangdong 525000, China \\ ${ }^{4}$ Nanning College of Technology, Guangxi 541006, China
}

(Received July 6, 2021; accepted October 19, 2021)

Keywords: MCU, LCD, infrared sensor, pulse signal

We aim to propose a simple design of a pulse meter. Pulse measurement is primarily based on a single-chip microcontroller unit (MCU) that receives the signal of a fingertip pulse and converts it into electrical signals using an infrared sensor. The signals are amplified and filtered by the proposed circuit and software. The pulse meter displays the measured value on a liquid crystal display (LCD) 1602 screen. The result with the prototype pulse meter with the proposed circuit design and software shows an accuracy of $98.9-102.5 \%$ and a relative standard deviation of $0.82-0.90 \%$. The proposed design provides the basis for developing further improved pulse meters that are appropriate for mobile devices.

\section{Introduction}

Pulse diagnosis is used in traditional Chinese medicine to diagnose patients' conditions by using three fingers (index, middle, and ring fingers) to detect the pulse on their wrist. It is known that pathological analysis is possible with pulse diagnosis. However, doctors may have different capabilities of diagnosing a patient's condition by feeling the pulse with their fingers depending on their experience and knowledge. Therefore, there are subjective factors that affect the accuracy of diagnosis. Thus, accurate pulse diagnosis requires modern technology to develop a more scientific and objective methodology than before. In modern medicine, heart rate and its variability are analogous to pulse diagnosis in traditional Chinese medicine. Heart rate variability (HRV) is an important physiological parameter for the clinical diagnosis of cardiovascular diseases, and research on HRV has been carried out in terms of medical, electronic, and engineering technology.

With the rapid improvement of the quality of life, people pay more attention to health than ever before, which leads to the development of cost-effective medical instruments for monitoring blood pressure, blood glucose, and so forth. They are appropriate for self-examination and selfrecording. There have been many research works on such medical instruments. Yang et al. ${ }^{(1)}$

\footnotetext{
*Corresponding author: e-mail: tslan888@yahoo.com.tw

** Corresponding author: e-mail: daixuanjun@163.com

https://doi.org/10.18494/SAM.2021.3537
} 
used a single-chip microcomputer with Arduino to measure and record an electrocardiogram (ECG), and sent the data to a LabVIEW master computer via a wireless network for calculation and display. This instrument uses ECG patches and piezoelectric pulse wave sensors instead of traditional inflatable cuffs, which measure blood pressure noninvasively. Peng et al. $^{(2)}$ designed a system of detecting pulse rate variability on the wrist, which could be used for daily monitoring. Chen et al. ${ }^{(3)}$ designed and implemented a health detection system based on an Android tablet, which can perform arrhythmia classification and heart rate variability analysis in real time. A portable oximeter system based on the MSP430 microcontroller was developed by He et $a l .{ }^{(4)}$. This system was characterized as low cost, small size, and easy to carry. Dabbakuti ${ }^{(5)}$ designed a low-power heart rate detection device based on a single programmable system on chip. Its algorithm allowed a detection rate of $1.59 \%$, and its average power consumption was only about $40.6 \mathrm{~W}$. These research works are for exploring and developing systems with portability, low energy consumption, high detection accuracy, and low cost.

However, to the best of our knowledge, there is no research on devices or systems that measure the pulse rate and blood pressure for diagnosis, because the development of these systems requires collecting physiological pulse signals and converting them into electrical signals. These are then calculated using an algorithm to determine whether the heart rate is normal. In this study, the system requires the use of infrared to measure the pulse of the human body. According to the difference in pulse during the relaxation and contraction of the heart, the intensity of the pulse is sensed as infrared signals by an infrared sensor. On the basis of this principle, we propose a new design of a pulse meter that requires measuring, counting, and displaying data, and other functions indicated in Fig. 1. The new design provides a pulse meter with the characteristics of stability and high precision as well as small size, easy to carry, compatibility with home equipment, and easy maintenance.

\section{Methods}

\subsection{Hardware structure}

The pulse meter includes an infrared sensor that converts the measured pulse signals into electrical ones and a single-chip control system for measurement and display. The meter includes a signal acquisition circuit, a signal processing circuit, buttons for selection, and a liquid crystal display (LCD) screen. The overall structure is shown in Fig. 2.

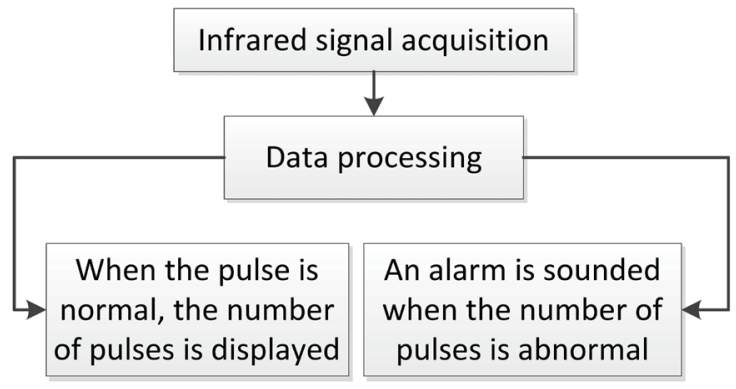

Fig. 1. Overall structure of a pulse meter. 


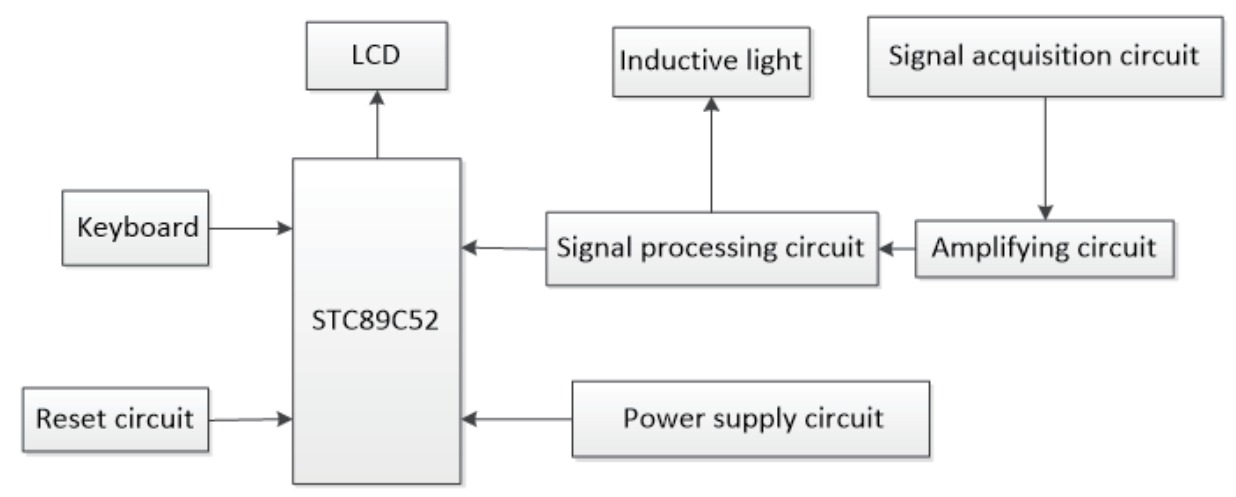

Fig. 2. Structure diagram of pulse meter.

\subsubsection{Control system}

\section{Microcontroller Unit (MCU)}

The pulse meter contains a microprocessor that is controlled by a program as the chip cannot be used alone. It relies on peripheral devices to perform properly. We selected an STC89C52 single-chip microcontroller, ${ }^{(6,7)}$ which consists of a central processing unit (CPU), data memory, program memory (ROM), a timer/counter, and I/O interfaces, as shown in Fig. 3.

\section{Circuit Design}

The control system of the pulse meter comprises a single-chip microcomputer, a crystal oscillator circuit, and a reset circuit, which function for inputs and outputs (display). The MCU in the pulse meter has low power consumption, small size, and easy adjustment. Figure 4 shows the circuit design of the MCU. The system's clock adopts the oscillating circuit with an external crystal of $12 \mathrm{MHz}$. A $10 \mathrm{~K}$ resistor is used as the pull-up resistor of port P0 with an open-drain structure. The MCU measures the pulse signal for $1 \mathrm{~min}$, processes it, and transmits the result to the display module.

\section{Sensor}

The pulse meter adopts an ST 188 sensor, the structure of which is shown in Fig. 5(a). The ST188 sensor consists of an infrared photodiode and a highly sensitive phototransistor. $A$ and $K$ are connected to an LED lamp, whereas $E$ and $C$ are connected to the emitter. According to the principle and internal structure of the reflective infrared photoelectric sensor, the circuit of the sensor is designed as shown in Fig. 5(b). With a peripheral circuit added to the sensor, the resistance is set as $R_{1}=330 \Omega$ and $R_{2}=20 \mathrm{k} \Omega$. When $A$ outputs ' 1 ' for a high-level voltage and $K$ outputs ' 0 ' for a low-level voltage, the infrared emission tube emits infrared rays. If the receiving tube receives the reflected infrared rays, the receiving head turns on, and $E$ outputs a high-level voltage, which is connected to $V_{c c}$. If the reflected infrared rays are not received, the infrared receiver does not turn on and $E$ outputs a low-level voltage and connects to the ground. The characteristics of the sensor are similar to those of a photoresistor. Depending on the intensity of the received infrared light, different voltages are generated by the sensor. When the voltage 


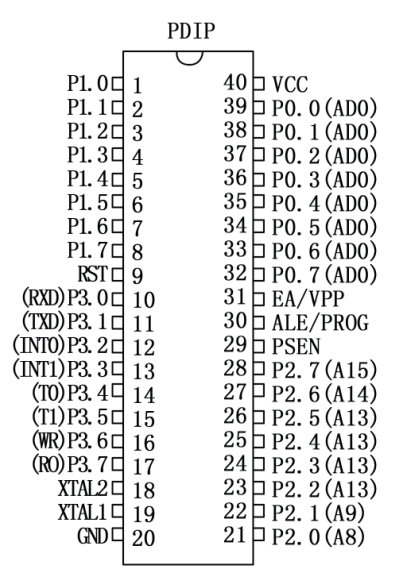

Fig. 3. Structure of MCU of pulse meter.

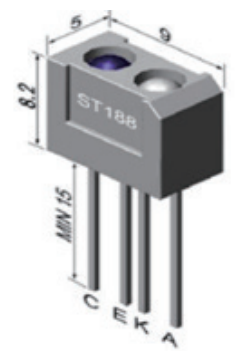

(a)

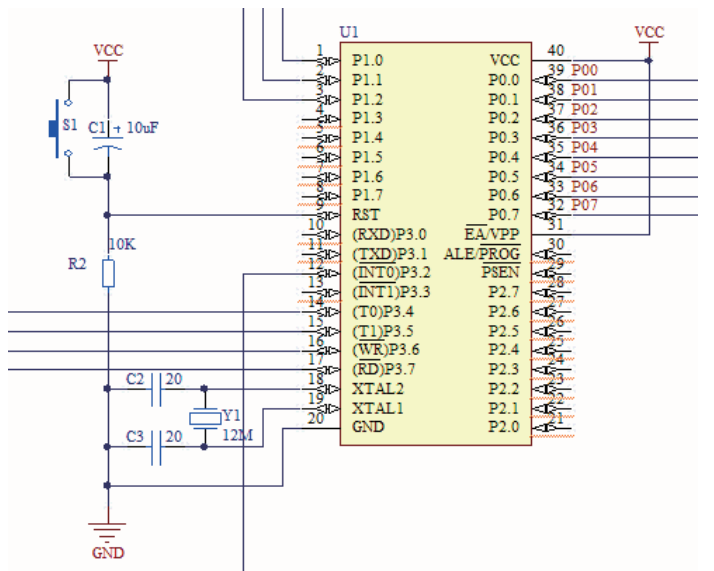

Fig. 4. (Color online) Circuit design of MCU.

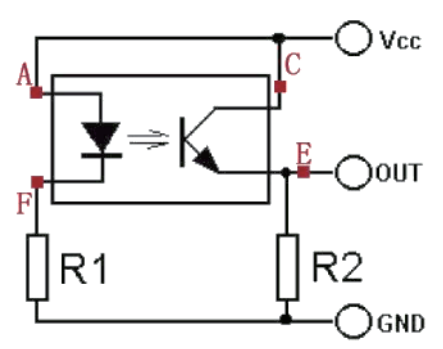

(b)

Fig. 5. (Color online) (a) Structure and (b) schematic diagram of ST188 sensor.

reaches a certain value, the triode will be in an amplified state, thereby generating different voltages.

\section{Signal Acquisition Circuit}

According to the working principle, ${ }^{(8)}$ the pulse is measured by the sensor. When the infrared ray passes through red blood cells, there is no reflection signal under non-hyperemia conditions. In this case, the signal-receiving head of the sensor does not turn on and then the voltage of $E$ is 0 . Under hyperemia conditions, the penetration ability of the infrared ray becomes weak, which enables $E$ to turn on to transmit a high output current. In this case, the electrical signal is generated in the circuit and transmitted to the next module. The circuit of the signal acquisition is shown in Fig. 6.

\subsubsection{Signal processing circuit}

\section{LM385 Amplifier}

The LM358 amplifier is a dual operational amplifier. It is composed of two independent highgain amplifiers that work under either a single or dual power supply. The LM358 amplifier can be used for all conventional operational amplifiers such as variable frequency amplifiers and DC 


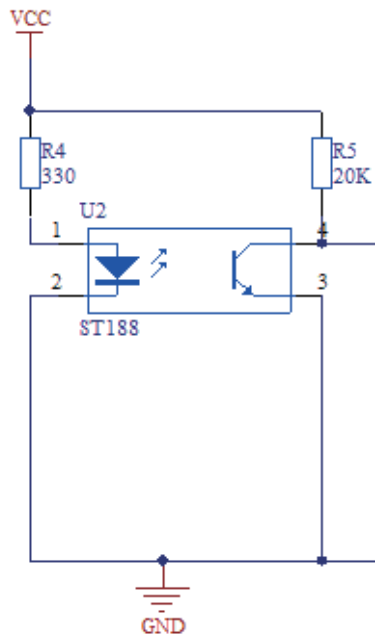

Fig. 6. (Color online) Signal acquisition circuit of pulse meter.

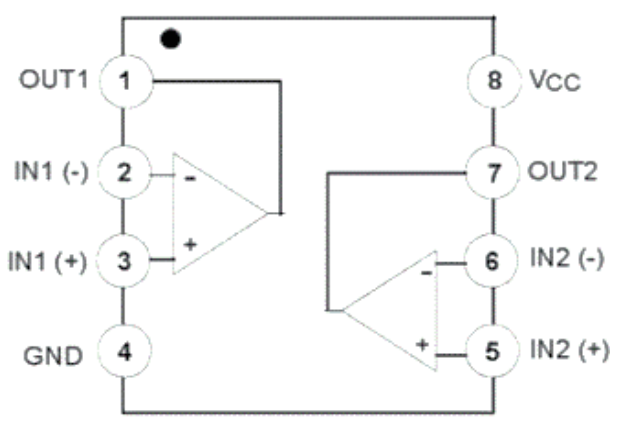

Fig. 7. Structure of LM358 amplifier.

gain components. It adopts the DIP8 or SOP8 package form. The pin diagram of the LM358 amplifier is shown in Fig. 7. Pin 8 is for the main power supply input, the voltages of pins 2 and 3 are compared and then output from pin 1 as OUT1. The voltages of pins 5 and 6 are compared and then output from pin 7 as OUT2. When IN1 $(+)>\operatorname{IN} 1(-)$ and IN2 $(+)>\operatorname{IN} 2(-)$, OUT1 and OUT2 output high-level voltages. If this condition is not met, a low-level voltage is output. The LM358 amplifier does not require a pull-up resistor, and the output voltage range is $-1.5-0 \mathrm{~V}$.

\section{Signal Processing Circuit}

Because the pulse signal is weak at the $\mu \mathrm{V}$ level and is usually accompanied by noise interference, it should be de-noised to make the signal clear. Even when the pulse signal is amplified and filtered, it still is irregular with low-frequency interference. As the irregularity does not meet the requirement for counting by the counter, a shaping circuit should be used to remove the irregularity. We adopt a hysteresis setting for a comparator (Fig. 8) to improve the anti-interference ability. With the LM358 amplifier, an LED light indicates the status of pulse beating.

\subsubsection{Display Circuit}

An LCD is used for thin and light displays owing to its physical, chemical, and optical properties. The main principle of the LCD is to stimulate the liquid crystal molecules with an electric current to generate dots, lines, and planes with a backlight. The LCD enables us to obtain high-quality images and small displays. We use the $\operatorname{LCD}^{(9)}$ with the circuit shown in Fig. 9. In the circuit, pins 7-14 are connected to the P0 port of the MCU for controlling the data display. Ports P1.2, P1.1, and P1.0 are used for enable (EN), read and write (R/W), and register selection (RS) of the LCD, respectively. The pulse signal is calculated by the MCU, and the result is transmitted to the display screen. 


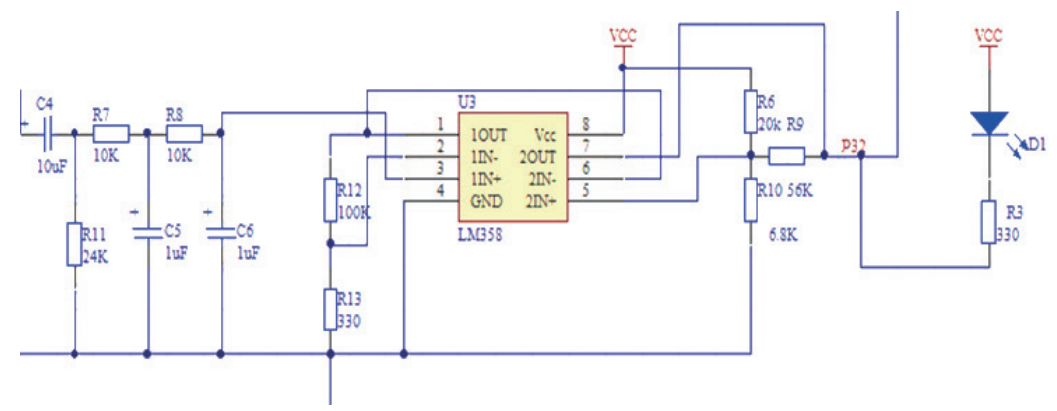

Fig. 8. (Color online) Circuit for signal processing.

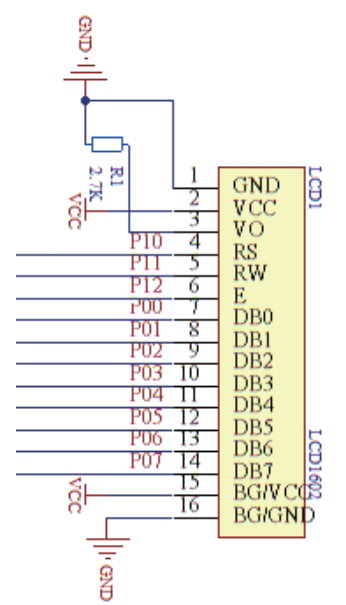

Fig. 9. (Color online) Data display circuit of pulse meter.

\section{Programming}

\subsection{Design of main program}

We use the Keil UV4 as a program compilation tool. ${ }^{(7,10)}$ Because the C language is more readable and convenient for expansion and transplantation than the assembler language, the program for the pulse meter is written in the $\mathrm{C}$ language. The program is initialized to realize the setting of special registers, timing interrupts, and various ports. The flow chart of the system software is shown in Fig. 10.

\subsection{Design of subroutine flow}

After the MCU is powered on, it initializes and clears the initial values of the previous parameters. Then, it waits for the user to press the corresponding button and enter the corresponding function. When the user presses the measurement button, the MCU measures the pulse rate for $15 \mathrm{~s}$, calculates the corresponding pulse rate, and then displays it on the LCD screen. When the user presses the pulse range setting button, the MCU increases or decreases the range depending on the user's requirements. 


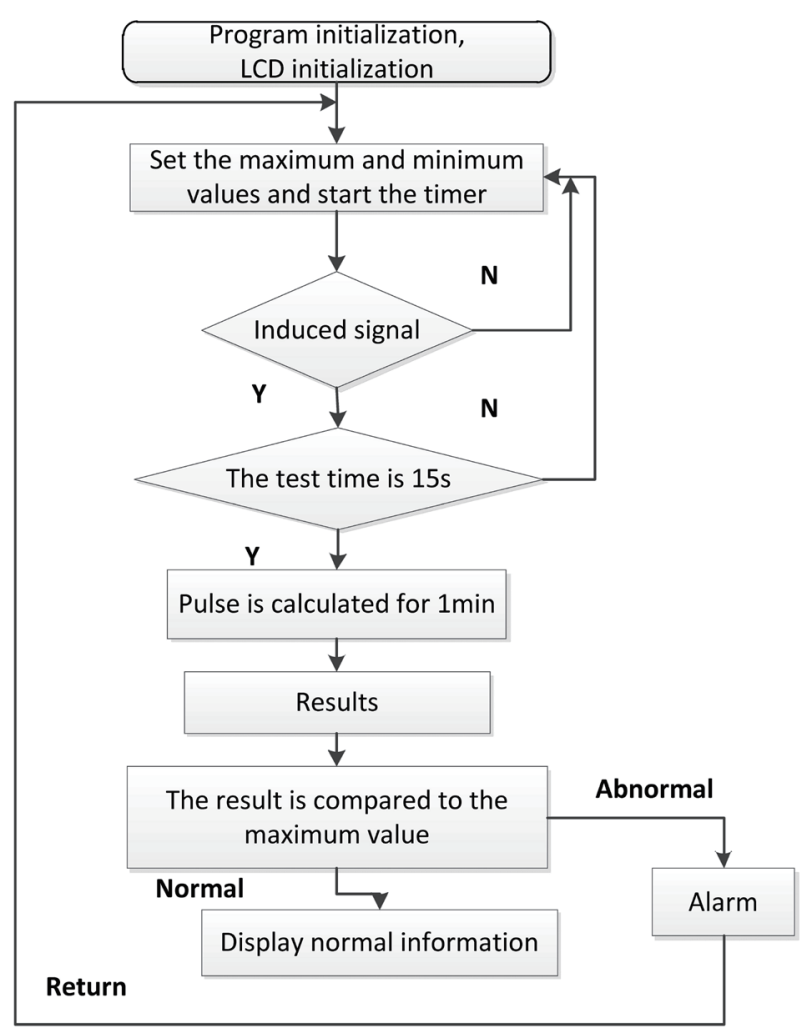

Fig. 10. Flow chart of software for pulse meter.

\section{Results and Discussion}

\subsection{Collection of pulse signals}

The human pulse is a weak low-frequency signal, so it should be amplified multiple times for the MCU to process the signal. The pulse of the human body is in the range of 50 to 200 beats per min with the frequency ranging from 0.83 to $3.33 \mathrm{~Hz}$. Therefore, the electrical signal measured by the infrared sensor is low. To minimize the error in detecting signals due to external high-frequency interference, the pulse signal must be low-pass-filtered to remove the high-frequency interference.

Then, for the stable operation of the pulse meter, the debugging of hardware and software is needed depending on the system design. Hardware debugging is mainly carried out to detect whether the short and open circuits have problems and to check the normal operation. Software debugging is mainly carried out to detect any signal input with timing interrupt. If there is no signal input, the software returns to the previous step where the interrupt is generated and the software is rechecked for debugging. After inspection and modification, the pulse meter becomes ready to operate normally. The prototype pulse meter manufactured in this study is shown in Fig. 12. 


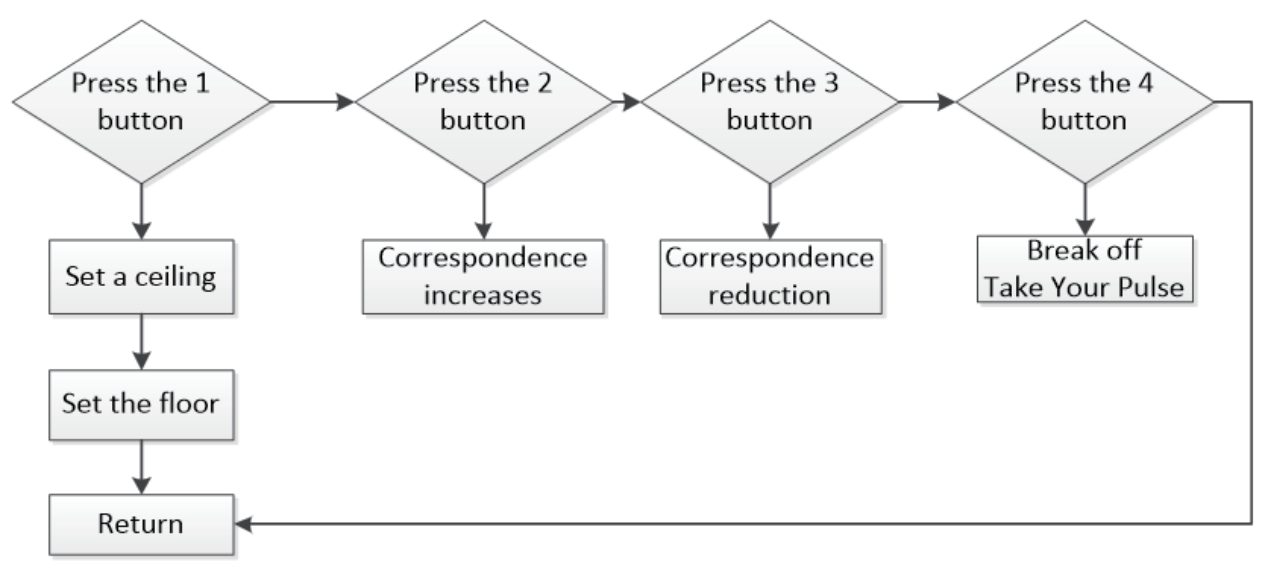

Fig. 11. Flow chart of subroutine of pulse meter.

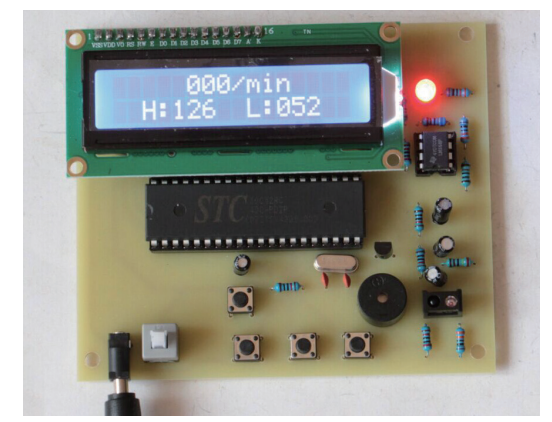

Fig. 12. (Color online) Image of prototype pulse meter.

Table 1

Measurement results.

\begin{tabular}{lccccc}
\hline \multirow{2}{*}{ User } & $\begin{array}{c}\text { Pulse on the wrist } \\
\text { (beats per minute) }\end{array}$ & \multicolumn{4}{c}{ Pulse of the finger (beats per minute) } \\
\cline { 2 - 6 } & 65 & 64 & 64 & 65 & Average $\left( \pm \mathrm{SD}^{*}\right)$ \\
\hline 1 & 72 & 70 & 71 & 71 & $64.3 \pm 0.577$ \\
2 & 68 & 69 & 70 & 70 & $69.7 \pm 0.577$ \\
3 & & & &
\end{tabular}

(*: Standard deviation)

The user should gently place a finger on the sensor to measure the pulse. As the pulse meter in this study has a highly sensitive sensor, the pulse of the finger can be detected. We measured the pulses on the wrists of three different users as reference values and used a pulse meter to measure the pulses of the fingers. The test results are shown in Table 1. These results show that the accuracy of the measurement compared with the pulse on the wrist ranges from 98.9 to $102.5 \%$, and the relative standard deviation (RSD) is $0.82-0.90 \%$. Thus, the pulse meter that measures the pulse of a finger had very accurate results when used to measure the pulse of the human body. 


\section{Conclusions}

In this study, we propose a new design of a pulse meter by using an STC89C52 microcontroller. The design is proposed for the effective amplification, filtering, and reshaping of the pulse signal and its effective processing by the MCU. The pulse signal is measured by an infrared sensor. The experiment result with the prototype pulse meter shows an accuracy of 98.9-102.5\% and an RSD of $0.82-0.90 \%$. The proposed design and measurement result with the pulse meter provide a foundation for developing a pulse meter for accurate pulse diagnosis in traditional Chinese medicine. The MCU proposed in this study is expected to be a basic one for further development of a similar pulse meter. The simple, efficient, accurate, and low-cost pulse meter is realized for accurate pulse measurement using the design proposed in this study.

\section{References}

1 B. Yang, Z. Z. Peng, and L. Xing: J. Biomed. Eng. Res. 38 (2019) 307. https://doi.org/10.19529/j.cnki.16726278.2019.03.09

2 R.-C. Peng, X.-L. Zhou, W.-H. Lin, and Y.-T. Zhang: Compt. Math. Methods Med. 2015 (2015) 1. https://doi. org $/ 10.1155 / 2015 / 516826$

3 X. Chen, X. Bao, F, Zhen, and S. Xia: Proc. 2016 IEEE Int. Conf. Information and Automation (ICIA, 2016) 1165-1168. https://doi.org/10.1109/ICInfA.2016.7831995

4 D. He, S. P. Morgan, D. Trachanis, J. V. Hese, D. Drogoudis, F. Fummi, F. Stefanni, V. Guarnieri, and B. R. Hayes-Gill: Sensors 15 (2015) 17076. https://doi.org/10.3390/s150717076

5 J. R. K. Kumar Dabbakuti: IJRTE 1 (2012) 59. https://www.researchgate.net/publication/301594241_Design and_Implementation_of_Portable_Health_Monitoring_system_using_PSoC_Mixed_Signal_Array_chip

6 Electronic Engineering and Product World: https:/global.cnki.net/KCMS/detail/detail.aspx?dbcode=CJFQ\&d bname $=$ CJFD2000\&filename $=$ DZCS200001024\&v $=$ MDAwNDFyQ1VSN3VmYnVSckZpSGdVci9JSVRmSW ZiRzRIdEhNcm85SF1JUjhlWDFMdXhZUzdEaDFUM3FUcldNMUY= (accessed September 2021).

7 X. Wang and J. Zhu: J. Beijing Inf. Sci. Technol. Univ. 31 (2016) 67. https://doi.org/0.16508/j.cnki.11$\underline{5866 / n .2016 .04 .013}$

8 B. Zhou, X. Wang, Q. Li, C. Qui, J, He, Y. Zhu, and H. Wang: J. Phys. Conf. Ser. 1924 (2021) 1. https://doi. org/10.1088/1742-6596/1924/1/012019

9 J. He and S. Y. Yuan: Adv. Mat. Res. 711 (2013) 598. https://doi.org/10.4028/www.scientific.net/AMR.711.598

10 M. Liu and X. Li: Electron. Test. 15 (2021) 21. https://doi.org/10.16520/j.cnki.1000-8519.2021.15.006 